\title{
BMJ Open Association of diabetic retinopathy and diabetic macular oedema with renal function in southern Chinese patients with type 2 diabetes mellitus: a single- centre observational study
}

\author{
Xuenan Zhuang, ${ }^{1,2}$ Dan Cao, ${ }^{1}$ Dawei Yang, ${ }^{1,2}$ Yunkao Zeng, ${ }^{1,2}$ Honghua Yu, ${ }^{1}$ \\ Jun Wang, ${ }^{1}$ Jian Kuang, ${ }^{3}$ Jianteng Xie, ${ }^{4}$ Shuting Zhang, ${ }^{3}$ Liang Zhang ${ }^{\oplus 1}$
}

To cite: Zhuang $X$, Cao $D$, Yang $\mathrm{D}$, et al. Association of diabetic retinopathy and diabetic macular oedema with renal function in southern Chinese patients with type 2 diabetes mellitus: a single-centre observational study. BMJ Open 2019;9:e031194. doi:10.1136/ bmjopen-2019-031194

\section{- Prepublication history for} this paper is available online. To view these files, please visit the journal online (http://dx.doi. org/10.1136/bmjopen-2017031194).

$\mathrm{XZ}$ and DC contributed equally.

Received 23 April 2019

Revised 01 August 2019

Accepted 02 August 2019

\section{Check for updates}

(C) Author(s) (or their employer(s)) 2019. Re-use permitted under CC BY-NC. No commercial re-use. See rights and permissions. Published by BMJ.

${ }^{1}$ Department of Ophthalmology, Guangdong Provincial People's Hospital, Guangzhou, China ${ }^{2}$ Shantou University Medical College, Shantou, China ${ }^{3}$ Department of Endocrinology, Guangdong Provincial People's Hospital, Guangzhou, China ${ }^{4}$ Department of Nephrology, Guangdong Provincial People's Hospital, Guangzhou, China

Correspondence to

Dr Liang Zhang;

zhangliang5413@163.com

\section{ABSTRACT}

Background and objectives The association of diabetic retinopathy (DR) and diabetic macular oedema (DME) with renal function in southern Chinese patients with diabetes is poorly understood. So we aimed to study the correlation between stage of DR and DME with stage of estimated glomerular filtration rate (eGFR) and stage of urine albumin-to-creatinine ratio (UACR), and to explore the systemic risk factors for DR and DME.

Design and setting This single-centre retrospective observational study was conducted from December 2017 to November 2018.

Participants 413 southern Chinese patients with type 2 diabetes mellitus.

Outcome measures The correlations between stage of DR and DME with stage of eGFR/UACR were assessed by Spearman's or $\chi^{2}$ analyses and represented with histograms. Risk factors associated with the occurrence of DR and DME were performed by logistic regression and represented with nomograms.

Results Stage of DR had a positive correlation with stage of eGFR $(r=0.264, p<0.001)$ and stage of UACR $(r=0.542$, $p<0.001)$. With the stage of eGFR/UACR being more severe, the prevalence of DME became higher as well (both $\mathrm{p}<0.001)$. The risk factors for DR were DM duration (OR 1.072; $95 \% \mathrm{Cl} 1.032$ to $1.114 ; p<0.001$ ), stage of UACR (OR 2.001; 95\% Cl 1.567 to 2.555; $\mathrm{p}<0.001)$ and low-density lipoprotein (LDL) (OR 1.301; 95\% Cl 1.139 to $1.485 ; p<0.001)$, while risk factors for DME were stage of UACR (OR 2.308; 95\% Cl 1.815 to $2.934 ; \mathrm{p}<0.001$ ) and LDL (OR 1.460; 95\% Cl 1.123 to $1.875 ; p=0.008$ ).

Conclusions Among southern Chinese patients, stage of $\mathrm{DR}$ and DME were positively correlated with renal function, while stage of UACR performed a better relevance than stage of eGFR.

\section{INTRODUCTION}

Diabetic retinopathy (DR) is a major global health burden owing to its high prevalence and associated high risk of vision loss. According to WHO, DR leads to $4.8 \%$ of the amount of cases of 37 million blindness
Strengths and limitations of this study

- The present study identified risk factors of diabetic retinopathy (DR) and diabetic macular oedema (DME) in a group of patients with diabetes with comprehensive blood tests results, therefore many confounders can be corrected in the logistic regression analyses.

- We developed nomograms for prediction DR and DME.

- Using these inexpensive, convenient, fast and intuitive tools, it will be more efficient for physicians to identify the patients with type 2 diabetes mellitus who are at a higher risk of DR and DME.

- This is a retrospective study; the findings in our study needs to be corroborated by prospective studies and randomised controlled trials.

The number of the cases in our study is small; it is better to enlarge the number of the participants.

around the world. ${ }^{1}$ China has a high prevalence of diabetes, with up to 113.9 million adults with diabetes and 493.4 million with prediabetes. ${ }^{2}$ The high prevalence of diabetes would be translated into an increase in the prevalence of diabetes complications. However, DR is always asymptomatic until it comes to advanced stages or occurrence of diabetic macular oedema (DME) leading to vision loss. Timely and reduplicative screening of DR can slow down its development, but the huge number of patients with diabetes and lack of ophthalmologists hampers frequent screening, especially in China. ${ }^{3}$ Therefore, how to screen DR more efficiently becomes an important issue in today's medical environment.

Chronic kidney disease (CKD), also known as one of the most common complication of diabetes, has similar pathogenesis with $\mathrm{DR}^{4}$ 
Reduced estimated glomerular filtration rate (eGFR) and elevated urine albumin-to-creatinine ratio (UACR) can be clinically used to evaluate renal function. ${ }^{5}$ Recent studies also found some relationships between UACR and eGFR with DR and DME. ${ }^{6-9}$ However, there is relatively few data on the relationship between stage of eGFR/ UACR and DR/DME in southern Chinese patients with type 2 diabetes mellitus (T2DM).

In this study, we tried to identify the association of DR/ DME with CKD in southern Chinese population. Moreover, we aim to identify potential systemic risk factors that are independently associated with DR/DME. Finally, risk factors for DR/DME recognised in the research were combined to make a nomogram, providing a novel and efficient screening tool for patients with T2DM who are at risk of DR and DME.

\section{METHOD}

\section{Study population}

This retrospective cross-sectional study was conducted on 413 patients in Department of Endocrinology of Guangdong Provincial People's Hospital who had undergone ophthalmic consultation from December 2017 to November 2018. Patients (aged $\geq 18$ years) with T2DM (according to the WHO criteria) ${ }^{10}$ and records of the Early Treatment Diabetic Retinopathy Study (ETDRS) 35-degree 7-standard fields colour retinal photographs (Topcon TRC; Topcon, Tokyo, Japan) were included in this study. Cases with any other ocular illness that possibly affect ocular circulation (eg, glaucoma, endophthalmitis, retinal vascular occlusion, age-related macular degeneration, refractive error $>3$ diopters, eye trauma), any severe systemic diseases (eg, myocardial infarction, cerebral infarction, connective tissue disorder) or history of any previous intravitreal injection or dialysis were excluded. Women who were pregnant or menstruating were also excluded. This study was performed according to the Declaration of Helsinki.

\section{Data collection}

All the medical information were acquired from the medical record. Demographic and physical data included gender, age, duration of diabetes mellitus (DM), height, weight and blood pressure. Body mass index was calculated as weight divided by height squared. Hypertension is defined as systolic blood pressure $\geq 140 \mathrm{~mm} \mathrm{Hg}$ or diastolic blood pressure $\geq 90 \mathrm{~mm} \mathrm{Hg}$. Laboratory tests included glycated haemoglobin, renal function tests (included serum nitrogen urea, serum creatinine $(\mathrm{Scr})$, urinary protein, urinary albumin and urinary creatinine (Ucr)), blood lipid (included non-estesterified fatty acid, high-density lipoprotein, low-density lipoprotein (LDL), triglyceride, total cholesterol (CHOL), lipoprotein a, apolipoprotein A (APOA) and apolipoprotein B (APOB)) and other blood biochemical indexes (included uric acid, alanine aminotransferase, aspartate transaminase, acetylcholinesterase, serum albumin, total protein, D-dimer and vitamin $\mathrm{B}_{12}$ ). Overnight fasting blood samples and urine samples were collected before 08:00 hours.

\section{Assessment of DR and DME}

DR and DME were diagnosed both on the clinical ophthalmic examination (slit lamp examination) and image evaluation (fundus photograph) by trained graders who were unaware of the medical conditions and further confirmed by a fundus expert (LZ) if two graders held different views. According to the International Clinical Diabetic Retinopathy and Diabetic Macular Oedema Disease Severity Scales, ${ }^{11}$ cases were divided into five groups for stage of DR: DR1/noDR, DR2/mild non-proliferative diabetic retinopathy (NPDR), DR3/moderate NPDR, DR4/severe NPDR and DR5/proliferative diabetic retinopathy (PDR). Among them, patient with DR was defined as DR2-5. In addition, DME was defined according to the ETDRS report: any retinal thickening or hard exudates within one disc diameter from the centre of foveal in the presence of DR features. ${ }^{12}$ Cases were also divided into two groups: patients without DME and patients with DME.

\section{Definition of stage of eGFR and stage of UACR}

Clinically, eGFR $\left(\mathrm{mL} / \mathrm{min} / 1.73 \mathrm{~m}^{2}\right)$ and UACR $(\mathrm{mg} / \mathrm{g})$ are always used to evaluate and manage kidney disease. The value of eGFR was calculated using the CKD-EPI creatinine equation ${ }^{13}$ as follows: $141 \times \min (\mathrm{Scr} / \kappa, 1)^{\alpha} \times \max$ $(\mathrm{Scr} / \kappa, 1)^{-1.209} \times 0.993^{\text {age }} \times 1.018$ (if female) $\times 1.159$ (if black), among which Scr is measured in $\mathrm{mg} / \mathrm{dL}, \kappa$ is 0.7 for females and 0.9 for males, $\alpha$ is -0.329 for females and -0.411 for males, min indicates the minimum of $\mathrm{Scr} / \mathrm{K}$ or 1 and max indicates the maximum of $\mathrm{Scr} / \kappa$ or 1 .

In our study, cases were divided into five groups (five stages of eGFR) according to the level of eGFR: eGFR1/ stage 1 of eGFR $\left(e G F R \geq 90 \mathrm{~mL} / \mathrm{min} / 1.73 \mathrm{~m}^{2}\right)$, eGFR2/ stage 2 of eGFR (eGFR $60-89 \mathrm{~mL} / \mathrm{min} / 1.73 \mathrm{~m}^{2}$ ), eGFR3/ stage 3 of eGFR (eGFR $30-59 \mathrm{~mL} / \mathrm{min} / 1.73 \mathrm{~m}^{2}$ ), eGFR4/ stage 4 of eGFR (eGFR $15-29 \mathrm{~mL} / \mathrm{min} / 1.73 \mathrm{~m}^{2}$ ) and eGFR $5 /$ stage 5 of eGFR (eGFR $<15 \mathrm{~mL} / \mathrm{min} / 1.73 \mathrm{~m}^{2}$ ). Also, subjects were divided into another five groups (five stages of UACR) based on the definition of the USA National Kidney Foundation ${ }^{14}$ : normal albuminuria (UACR1/stage 1 of UACR, UACR $<10 \mathrm{mg} / \mathrm{g}$ ), low albuminuria (UACR2/stage 2 of UACR, UACR $10-29 \mathrm{mg} / \mathrm{g}$ ), microalbuminuria (UACR3/stage 3 of UACR, UACR 30-299 mg/g), macroalbuminuria (UACR4/stage 4 of UACR, UACR 300-999 mg/g) and severe macroalbuminuria (UACR 5 /stage 5 of UACR, UACR $\geq 1000 \mathrm{mg} / \mathrm{g}$ ).

\section{Statistical analysis}

Data analyses performed using SPSS software V.19.0 (SPSS, Chicago, Illinois, USA) and 'R Language' statistical package. Normality of the data was tested by using the Shapiro-Wilk test and inspecting the histograms. Continuous data were summarised as mean $\pm \mathrm{SD}$ or medians (IQRs), while discrete (categorical) in number and percentage (\%). Independent t-test and Mann-Whitney 
$\mathrm{U}$ test were used to compare normally distributed and non-normally distributed data, respectively; $\chi^{2}$ analysis was used to compare categorical data. Spearman's correlation coefficient was used to evaluate the correlation between stages of DR and stages of eGFR or UACR. In all statistical analyses, criterion significance was considered at the $\mathrm{p}<0.05$ level.

To make nomograms for prediction of the risk of DR and DME, we conducted a several-steps analysis of the relationship between the presence of $\mathrm{DR} / \mathrm{DME}$ and the risk factors. First, we carried out the univariate binary logistic regression analyses, in which we took the existence of DR or DME as the dependent variable and all risk factors (included four parts as following tables) as independent variables. Second, the variables, which were found statistically significant in the univariate analysis but not mutual interfere, were entered in the multivariate binary regression analyses model. For example, stage of UACR was calculated by urinary albumin and Ucr as well as the level of UACR; despite these four factors were all found statistically significant in the univariate analysis, we only chose the stage of UACR, which is calculated finally, into the multivariate analysis. Outcomes of these regression analyses were expressed as ORs, CI stated at $95 \%$ and $\mathrm{p}$ value. Third, nomograms for DR and DME risk factors were developed, ${ }^{15}$ and the risk factors which were statistically significant in former multivariate regression were selected as predictors. Fourth, receiver operating characteristic (ROC) analysis was carried out to determine the performance of these risk factors and nomograms. In these analyses, we treated the stage of eGFR and stage of UACR as continuous variables, but not categorical variables.

\section{Patient and public involvement}

Patients and the public were not directly involved in the design, conduct and reporting of our research.

\section{RESULTS}

A total of 413 patients (232 males and 181 females) were included in the study. The mean age of enrolled subjects was $58.74 \pm 13.75$ years. The mean duration of a T2DM diagnosis was $9.76 \pm 7.77$ years (range: $1-31$ years). One hundred and sixty participants had manifestations of DR, with a prevalence of $38.74 \%$. In detail, the percentage of mild, moderate, severe NPDR and PDR was $8.23 \%(\mathrm{n}=34)$, $16.22 \%(\mathrm{n}=67), 7.51 \%(\mathrm{n}=31)$ and $6.78 \%(\mathrm{n}=28)$, respectively. Fifty-three people had DME, with a prevalence of $12.83 \%$ in total 413 subjects and $33.13 \%$ in 160 DR subjects.

The stage of DR was positively associated with stage of eGFR and UACR (both $\mathrm{p}<0.001$ ), and stronger correlation was observed between stage of DR and stage of UACR $(r=0.264$ between stage of DR and stage of eGFR, $r=0.542$ between stage of DR and stage of UACR) (figure 1). The stage of eGFR and UACR also had correlation with DME development (both $\mathrm{p}<0.001$, figure 2 ). With the stage of eGFR or UACR being more severe, the prevalence of DME became higher as well.

Table 1 shows the clinical characteristics and the comparisons in the groups with DR and without DR. DM duration, hypertension, urinary protein, urinary albumin, UACR, urinary protein/Ucr (UPCR), LDL, CHOL, APOB and D-dimer were obviously increased significantly in group with DR compared with the groups without DR (all $\mathrm{p}<0.001$ ). In contrast, Ucr and ALB were decreased significantly in the DR group $(\mathrm{p}<0.001)$.

With respect to with/without DME groups (table 2), more renal function tests (included serum urea, Scr, urinary protein, urinary albumin, UACR and UPCR), LDL and D-dimer increased significantly in DME group compared with the group without DME (all $\mathrm{p}<0.001$ ). Inversely, Ucr, eGFR and ALB decreased significantly in the DME group $(\mathrm{p}<0.001)$.

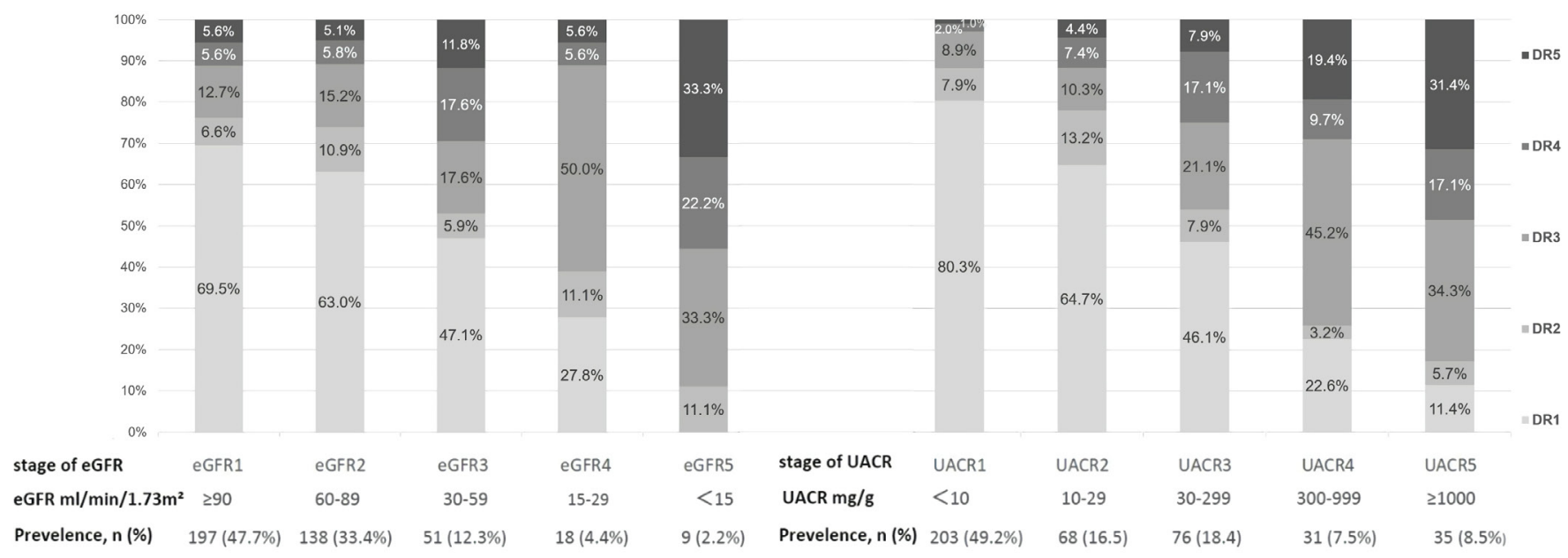

Figure 1 Correlation between stage of eGFR and UACR with stage of DR. The $n \%$ displayed in the histogram means the percentage of patients in each stacked bar plots. DR, diabetic retinopathy; eGFR, estimated glomerular filtration rate; UACR, urine albumin-to-creatinine ratio. 


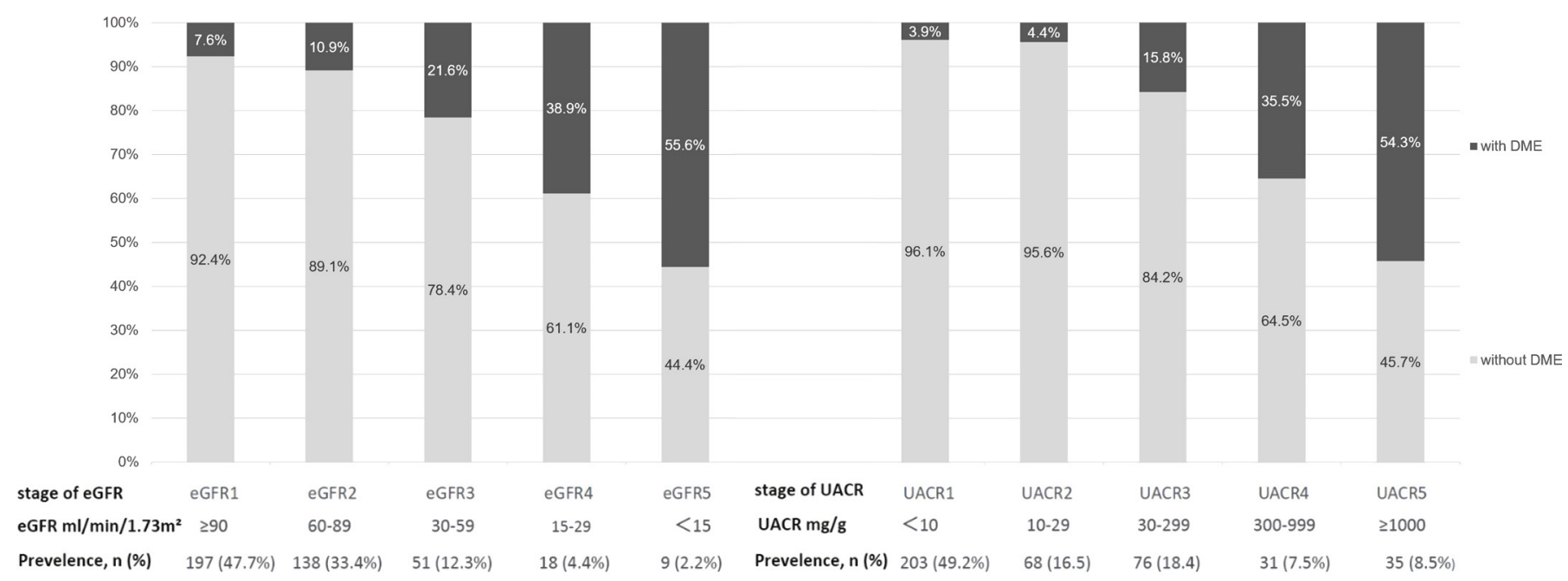

Figure 2 Correlation between stage of eGFR and UACR with occurrence of DME. The n\% displayed in the histogram means the percentage of patients in each stacked bar plots. DME, diabetic macular oedema; eGFR, estimated glomerular filtration rate; UACR, urine albumin-to-creatinine ratio.

Multivariate regression analyses (table 3) exhibited a positive relationship between the risk of DR with DM duration (OR 1.072; 95\% CI 1.032 to 1.114; $\mathrm{p}<0.001$ ), stage of UACR (as a continuous variable; OR 2.001; 95\% CI 1.567 to $2.555 ; \mathrm{p}<0.001$ ) and LDL (OR $1.301 ; 95 \%$ CI 1.139 to $1.485 ; \mathrm{p}<0.001$ ). The area under the curve (AUC) of stage of UACR to predict DR was 0.749 (95\% CI 0.639 to 0.741 ; $\mathrm{p}<0.001$ ), with higher than DM duration (AUC 0.690; $95 \%$ CI 0.699 to $0.800 ; \mathrm{p}<0.001$ ) and LDL (AUC 0.557; $95 \%$ CI 0.502 to $0.613 ; \mathrm{p}=0.042$ ).

Table 4 demonstrates a positive relationship between the risk of DME with stage of UACR (OR 2.308; 95\% CI 1.815 to $2.934 ; \mathrm{p}<0.001)$ and LDL (OR $1.460 ; 95 \%$ CI 1.123 to $1.875 ; \mathrm{p}=0.008)$. The AUC to predict DME of stage of UACR was 0.796 (95\% CI 0.726 to $0.867 ; \mathrm{p}<0.001)$, while that of LDL was 0.634 (95\% CI 0.548 to $0.720 ; \mathrm{p}=0.002$ ).

Based on the results of multivariate regression analyses, nomogram using three parameters (DM duration, stages of UACR and LDL) for risk of DR in people with T2DM are formulated in figure 3 , with a total predictive accuracy of $78.1 \%$ ( $95 \%$ CI $73.4 \%$ to $82.7 \%, p<0.001$ ). As shown in figure 4, nomogram for the prediction of DME, which had a ROC analysis revealing the overall predictive accuracy of $82.2 \%$ ( $95 \%$ CI $75.9 \%$ to $88.5 \%$; $\mathrm{p}<0.001$ ), was constructed using two parameters (stage of UACR and LDL).

\section{DISCUSSION}

Our study directly demonstrated association between stages of DR and presence of DME with stages of eGFR and UACR (figures 1 and 2) in southern Chinese patients. Besides, we developed a nomogram for prediction of DR and DME.

The Spearman's analyses revealed that stage of DR was correlated with stage of eGFR and UACR, while the former one had a lower correlation coefficient $(r=0.264, \mathrm{p}<0.001)$ than the latter one $(r=0.542, p<0.001)$. Besides, even after adjustment for other variables, the disease rate of DR increases in patients with T2DM with higher stage of UACR.
With respect to eGFR, another important marker for CKD, it showed poorer effect for the development of DR in multivariate logistic analysis (both $\mathrm{p}$ value of eGFR and stage of eGFR are $>0.05$ ). Presumably speaking, higher level of UACR is associated with more to the occurrence of DR than higher level of eGFR. This result is consistent with many previous studies. Sabanayagam et al found that DR was associated with CKD only in the appearance of albuminuria. ${ }^{6}$ Romero-Aroca et al found that UACR and eGFR are two risk factors for DME, but UACR being a more important risk factor. ${ }^{9}$ Chen et al demonstrated that UACR appeared to be more associated with the development and progression of DR compared with decline in eGFR among population with T2DM. ${ }^{16}$

Diabetic retinopathy shares analogous pathogenesis with diabetic kidney disease (DKD) ${ }^{4}$ including hyperglycaemia-induced oxidative stress, accumulation of advanced glycation end products, increased production of reactive oxygen species, abnormal activation of protein kinase $\mathrm{C}$, abnormal activation of the reniningiotensin system, etc. All these factors have resulted in a parallel manner of DR and DKD, manifested in UACR as one predictive factor for DR. Nevertheless, why eGFR acted worse than UACR in predicting DR? There might be two possible explanations. First, eGFR is elevated in early stages of DKD and declines with disease progression. Second, T2DM is more heterogeneous than type $1 \mathrm{DM}$. A third of patients with T2DM and renal insufficiency have no proteinuria. ${ }^{17}$ Therefore, the decline of eGFR in patients with T2DM may attribute to non-DKD nephropathy, arteriosclerosis or accelerated ageing of kidney, etc. ${ }^{4}$ Accordingly, and to be superimposed, these may be the reasons why decreased eGFR cannot be used as a predictor for DR.

In addition, as the $\chi^{2}$ analyses showed, with stages of eGFR or UACR being more severe, the prevalence of DME increased. However, the logistic regression analysis for DME indicated that only stage of UACR but no stage of eGFR 
Table 1 Characteristics of DR group and no DR group

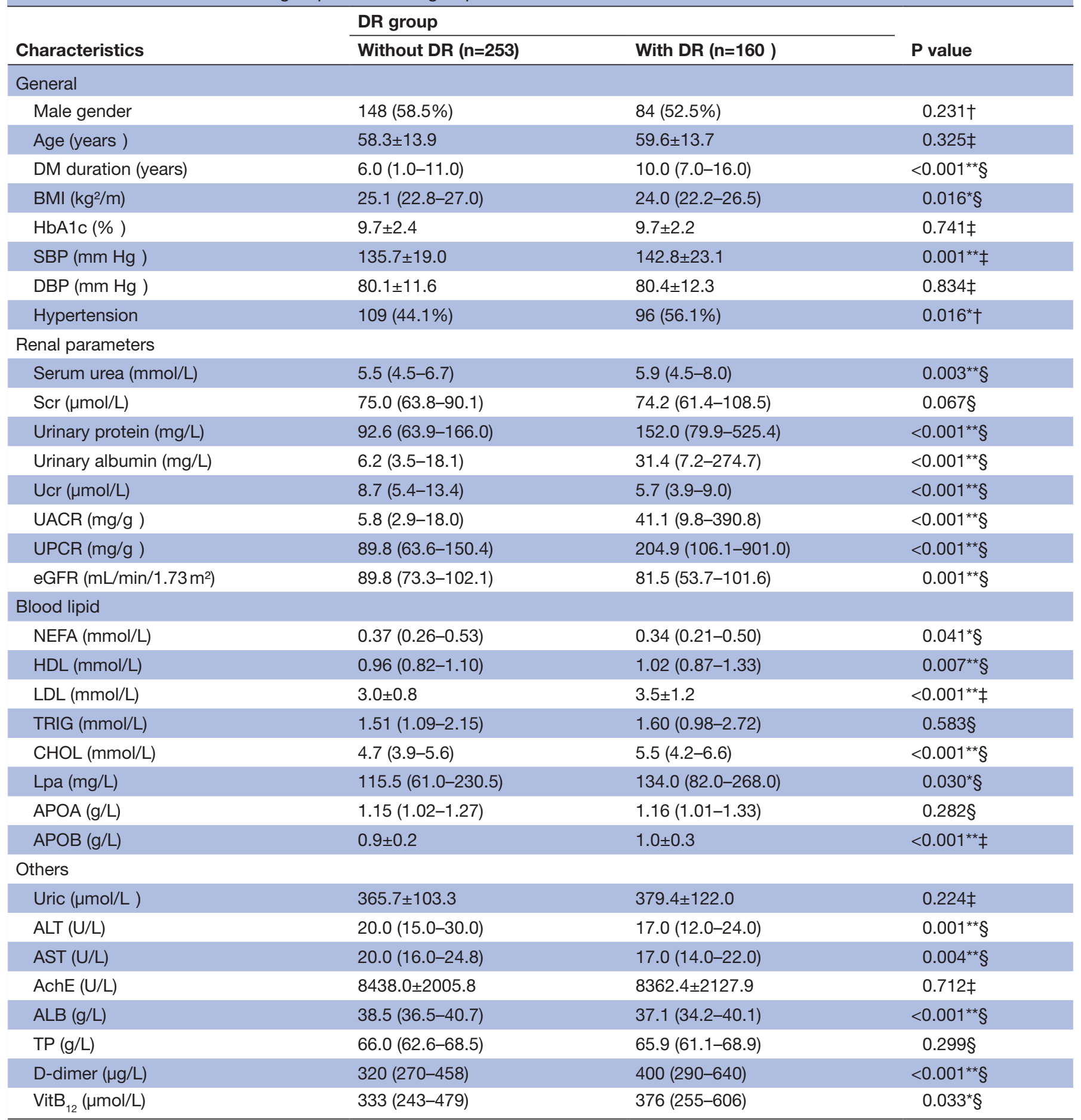

Results are expressed as mean $\pm S D$, percentages or as medians $(\mathrm{IQR})$; $\mathrm{p}$ values were compared by independent $\mathrm{t}$-test, Mann-Whitney $\mathrm{U}$ test or $\chi^{2}$ test as appropriate.

${ }^{*} \mathrm{P}<0.05,{ }^{* *} \mathrm{p}<0.01$.

†Values for comparisons between groups by $\chi^{2}$ test.

$\ddagger$ Values for comparisons between groups by independent samples t-test.

$\S$ Values for comparisons between groups by Mann-Whitney $U$ test.

AchE, acetylcholinesterase;ALB, serum albumin; ALT, alanine aminotransferase; APOA, apolipoprotein A; APOB, apolipoprotein B; AST, aspartate transaminase; BMI, body mass index; CHOL, total cholesterol; DBP, diastolic blood pressure; DM, diabetes mellitus; DR, diabetic retinopathy; eGFR, estimated glomerular filtration rate; HbA1c, glycated haemoglobin; HDL, high-density lipoprotein; LDL, lowdensity lipoprotein; Lpa, lipoproteina; NEFA, non-estesterified fatty acid; SBP, systolic blood pressure; Scr, serum creatinine; TP, total protein; TRIG, triglyceride; UACR, urine albumin-to-creatinine ratio; Ucr, urinary creatinine; UPCR, urinary protein/Ucr; VitB ${ }_{12}$, vitamin $B_{12}$. 
Table 2 Characteristics of DME group and no DME group

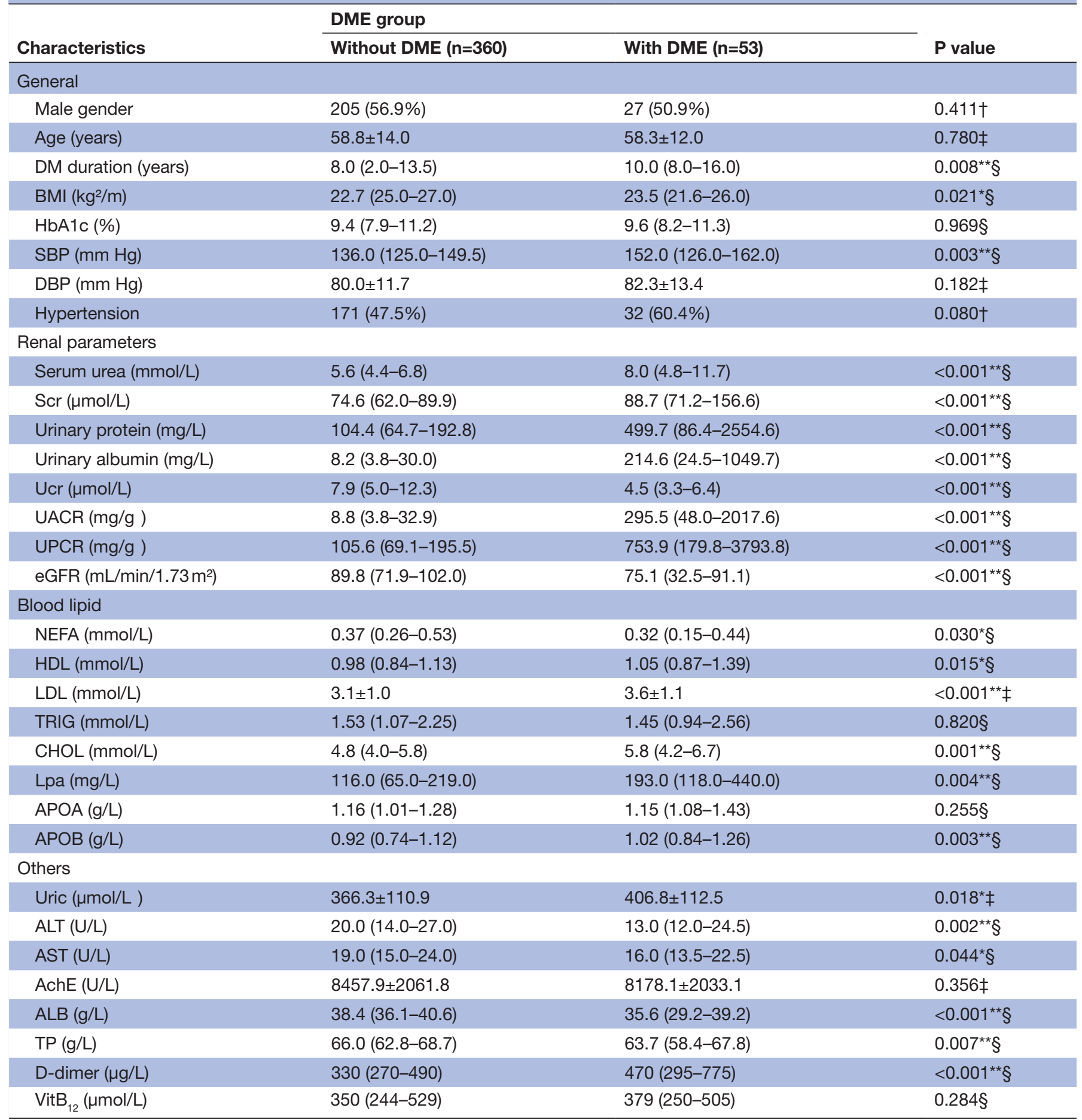

Results are expressed as mean $\pm \mathrm{SD}$, percentages or as medians (IQR); $\mathrm{p}$ values were compared by independent t-test, Mann-Whitney $\mathrm{U}$ test or $\chi^{2}$ test as appropriate.

${ }^{*} \mathrm{P}<0.05,{ }^{* *} \mathrm{p}<0.01$.

†Values for comparisons between groups by $\chi^{2}$ test.

$\ddagger$ Values for comparisons between groups by independent samples t-test.

$\S$ Values for comparisons between groups by Mann-Whitney U test.

AchE, serum acetylcholinesterase; ALB, serum albumin; ALT, alanine aminotransferase; APOA, apolipoprotein A; APOB, apolipoprotein B; AST, aspartate transaminase; BMI, body mass index; CHOL, total cholesterol; DBP, diastolic blood pressure; DM, diabetes mellitus; DME, diabetic macular oedema; DR, diabetic retinopathy; eGFR, estimated glomerular filtration rate; HbA1c, glycated hemoglobulin; HDL, high-density lipoprotein; LDL, low-density lipoprotein; Lpa, lipoprotein a; NEFA, non-estesterified fatty acid; SBP, systolic blood pressure; Scr, serum creatinine; TP, total protein; TRIG, triglyceride; UACR, urine albumin-to-creatinine ratio; Ucr, urinary creatinine; UPCR, urinary protein/Ucr; VitB ${ }_{12}$, vitamin $B_{12}$. 
Table 3 Risk factors for DR using binary logistic regression analysis

\begin{tabular}{|c|c|c|c|c|}
\hline \multirow[b]{2}{*}{ characteristics } & \multicolumn{2}{|l|}{ Univariate model } & \multicolumn{2}{|l|}{ Multivariate model } \\
\hline & OR (95\% Cl) & $P$ value & OR (95\% Cl) & $P$ value \\
\hline DM duration (per year) & $1.062(1.034$ to 1.091$)$ & $<0.001^{\star *}$ & $1.072(1.032$ to 1.114$)$ & $<0.001$ \\
\hline Hypertension & 1.621 (1.094 to 2.400$)$ & $0.016^{\star}$ & & \\
\hline SBP (per mm Hg ) & $1.016(1.007$ to 1.026$)$ & $0.001^{\star \star}$ & & \\
\hline Urinary protein (per mg/L) & 1.001 (1.000 to 1.001$)$ & $<0.001^{\star \star}$ & & \\
\hline Urinary albumin (per mg/L) & $1.002(1.001$ to 1.002$)$ & $<0.001^{\star \star}$ & & \\
\hline Ucr (per $\mu \mathrm{mol} / \mathrm{L})$ & 0.948 (0.913 to 0.984$)$ & $0.005^{\star \star}$ & & \\
\hline UACR (per mg/g ) & $1.001(1.000$ to 1.001$)$ & $<0.001^{\star *}$ & & \\
\hline UPCR (per mg/g ) & $1.000(1.000$ to 1.000$)$ & $<0.001^{\star \star}$ & & \\
\hline HDL (per mmol/L) & 2.364 (1.261 to 4.433$)$ & $0.007^{\star \star}$ & & \\
\hline LDL (per mmol/L) & $1.566(1.274$ to 1.925$)$ & $<0.001^{\star \star}$ & 1.301 (1.139 to 1.485$)$ & $<0.001^{\star \star}$ \\
\hline CHOL (per mmol/L) & $1.299(1.137$ to 1.483$)$ & $<0.001^{\star \star}$ & & \\
\hline APOB (per g/L) & 4.108 (1.919 to 8.792$)$ & $<0.001^{\star \star}$ & & \\
\hline ALT (per U/L) & 0.982 (0.968 to 0.996$)$ & $0.010^{\star}$ & & \\
\hline AST (per U/L) & 0.976 (0.957 to 0.996$)$ & $0.018^{*}$ & & \\
\hline ALB (per g/L) & 0.921 (0.882 to 0.962$)$ & $<0.001^{\star \star}$ & & \\
\hline D-dimer (per $\mu g / L)$ & $1.001(1.000$ to 1.001$)$ & $0.003^{\star \star}$ & & \\
\hline
\end{tabular}

Values express as ORs, $\mathrm{Cl}$ stated at $95 \%$ and $\mathrm{p}$ value.

All parameters with $\mathrm{p} \geq 0.05$ were not displayed in this table.

${ }^{*} \mathrm{P}<0.05,{ }^{* *} \mathrm{p}<0.01$.

ALB, serum albumin; ALT, alanine aminotransferase; APOB, apolipoprotein B; AST, aspartate transaminase; BMI, body mass index; CHOL, total cholesterol; CKD, chronic kidney disease; DM, diabetes mellitus; DR, diabetic retinopathy; eGFR, estimated glomerular filtration rate; HDL, high-density lipoprotein; LDL, low-density lipoprotein; SBP, systolic blood pressure; UACR, urine albumin-to-creatinine ratio; Ucr, urinary creatinine; UPCR, urinary protein/Ucr.

was a predictor for DME, which was similar with the result of DR's.

A common pathophysiological mechanism, vascular hyperpermeability, possibly occur both in DME and albuminuria (high UACR). Damage of the blood-retinal barrier in DR results in leakage of lipoproteins from the retinal capillaries to the retinal extravascular space, which results in DME. ${ }^{18}$ Analogously, breakdown of the glomerular filtration barrier in DKD results in high UACR. ${ }^{19} 20$ This may be the reason why stage of UACR could act as a predictor for DME.

Besides the stage of UACR as risk factors for predicting DR and DME, there were several other risk factors recognised in the logistic regression analyses: DM duration and LDL for DR, and LDL for DME. DM duration had been found as the risk factor in prediction of DR in many previous studies. ${ }^{21} 22$ As for LDL, there were several studies ${ }^{23-26}$ found associations between high level of LDL with DR/DME, which had similar results with our study.
In a recent study, Kang et al even found that patients with diabetes taking statins were $14 \%$ less likely to develop retinopathy than those not taking statins. ${ }^{27}$ These indicated dyslipidemia (high level of LDL) do harm to DR and DME, and statin therapy (reducing LDL and other serum lipids) may delay the progression of DR. Du et al demonstrated that increased levels of LDL might promote retinal-blood barrier injury through endoplasmic reticulum stress, apoptosis, oxidative stress and autophagy in patients with DR. ${ }^{28}$ These can perhaps explain why LDL exhibited as the risk factors for both DR and DME.

Nomogram, a graph of complex mathematical formulas, $^{15}$ is widely used for prognosis of tumours. However, few studies have described this manoeuvrable tool for DR risk prediction. ${ }^{29}$ In the final part of this paper, we developed nomograms for prediction of DR and DME. By means of the nomograms, doctors can estimate the probability of developing DR or DME in a single patient. Taking a patient with stage 4 of UACR and LDL 
Table 4 Risk factors for DME using binary logistic regression analysis

\begin{tabular}{|c|c|c|c|c|}
\hline \multirow[b]{2}{*}{ Characteristics } & \multicolumn{2}{|l|}{ Univariate model } & \multicolumn{2}{|l|}{ Multivariate model } \\
\hline & OR $(95 \% \mathrm{Cl})$ & $P$ value & OR $(95 \% \mathrm{Cl})$ & $P$ value \\
\hline DM duration (per year) & 1.036 (1.001 to 1.072$)$ & $0.044^{*}$ & & \\
\hline BMI (per kg²/m) & 0.914 (0.838 to 0.997$)$ & $0.042^{*}$ & & \\
\hline SBP (per mm Hg ) & 1.023 (1.009 to 1.037$)$ & $0.001^{* *}$ & & \\
\hline Serum urea (per mmol/L) & 1.162 (1.086 to 1.244$)$ & $<0.001^{\star \star}$ & & \\
\hline Scr $($ per $\mu \mathrm{mol} / \mathrm{L})$ & 1.009 (1.005 to 1.012$)$ & $<0.001^{* *}$ & & \\
\hline Urinary protein (per mg/L) & 1.001 (1.000 to 1.001$)$ & $<0.001^{\star *}$ & & \\
\hline Urinary albumin (per mg/L) & 1.001 (1.001 to 1.002$)$ & $<0.001^{\star *}$ & & \\
\hline Ucr (per $\mu \mathrm{mol} / \mathrm{L})$ & 0.839 (0.770 to 0.914$)$ & $<0.001^{\star \star}$ & & \\
\hline UACR (per mg/g ) & 1.001 (1.001 to 1.001$)$ & $<0.001^{\star \star}$ & & \\
\hline UPCR (per mg/g ) & $1.000(1.000$ to 1.000$)$ & $<0.001^{\star \star}$ & & \\
\hline eGFR (per $\mathrm{mL} / \mathrm{min} / 1.73 \mathrm{~m}^{2}$ ) & 0.975 (0.965 to 0.985$)$ & $<0.001^{\star \star}$ & & \\
\hline Stage of UACR (per stage) & 2.449 (1.937 to 3.096$)$ & $<0.001^{\star \star}$ & 2.308 (1.815 to 2.934$)$ & $<0.001^{* *}$ \\
\hline Stage of CKD (per stage) & 1.976 (1.516 to 2.574$)$ & $<0.001^{* *}$ & & \\
\hline Uric (per $\mu \mathrm{mol} / \mathrm{L}$ ) & 1.003 (1.001 to 1.006$)$ & $0.015^{\star}$ & & \\
\hline $\mathrm{HDL}$ (per mmol/L) & 2.416 (1.143 to 5.107$)$ & $0.021^{*}$ & & \\
\hline LDL (per mmol/L) & 1.605 (1.212 to 2.124$)$ & $0.001^{* *}$ & $1.460(1.123$ to 1.875$)$ & $0.008^{\star *}$ \\
\hline $\mathrm{CHOL}$ (per mmol/L) & 1.252 (1.069 to 1.467$)$ & $0.005^{\star *}$ & & \\
\hline Lpa (per mg/L) & 1.001 (1.000 to 1.002$)$ & $0.034^{*}$ & & \\
\hline APOB (per g/L) & $4.849(1.705$ to 13.790$)$ & $0.003^{* *}$ & & \\
\hline ALB (per g/L) & 0.875 (0.827 to 0.925$)$ & $<0.001^{\star \star}$ & & \\
\hline TP (per g/L) & 0.940 (0.897 to 0.984$)$ & $0.009^{* *}$ & & \\
\hline
\end{tabular}

Values express as ORs, $\mathrm{Cl}$ stated at $95 \%$ and $\mathrm{p}$ value.

All parameters with $\mathrm{p} \geq 0.05$ were not displayed in this table.

${ }^{*} \mathrm{P}<0.05,{ }^{* *} \mathrm{p}<0.01$.

ALB, serum albumin; APOB, apolipoprotein B; BMI, body mass index; CHOL, total cholesterol; CKD, chronic kidney disease; DM, diabetes mellitus; eGFR, estimated glomerular filtration rate; HDL, high-density lipoprotein; LDL, low-density lipoprotein; Lpa, lipoprotein a; SBP, systolic blood pressure; Scr, serum creatinine; TP, total protein; UACR, urine albumin-to-creatinine ratio; Ucr, urinary creatinine; UPCR, urinary protein/Ucr.

level of $5 \mathrm{mmol} / \mathrm{L}$ along with DM duration for 20 years vertically to the first row for a certain point: DM duration as an example, the interpretation of these nomograms for 20 years points to 33 points, stage 4 of UACR points to includes three main parts-first, link the predictor's value $\quad 70$ points and LDL level of $5 \mathrm{mmol} / \mathrm{L}$ points to 34 points;

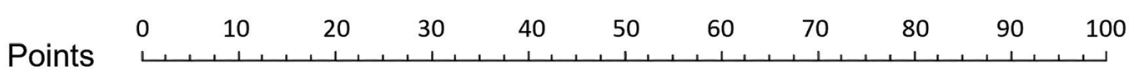

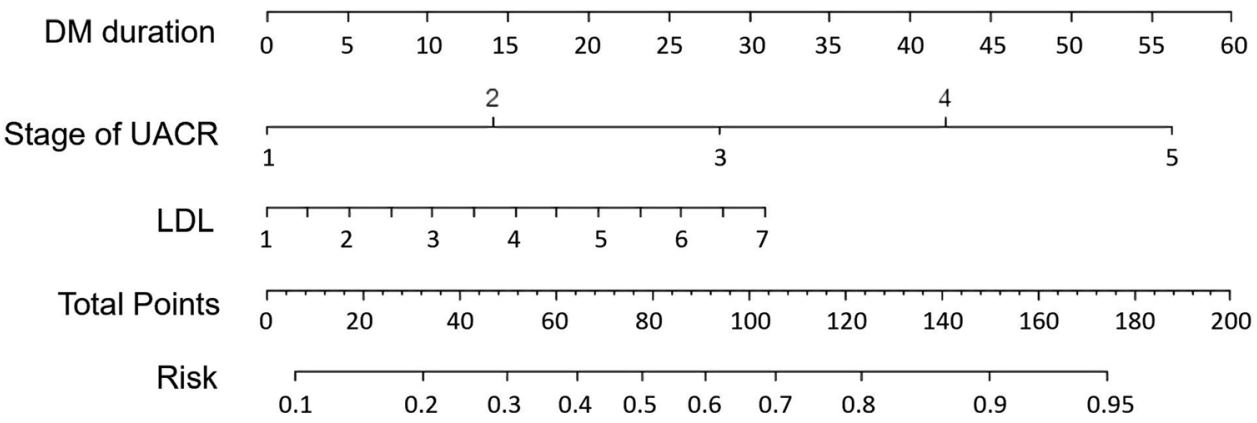

Figure 3 Nomogram to define the risk for the occurrence of DR. This nomogram was developed according to the statistically significant risk factors (DM duration, stage of UACR and LDL) (AUC 0.781; 95\% Cl 0.734 to $0.827 ; p<0.001$ ). AUC, area under the curve; DM, diabetes mellitus; DR, diabetic retinopathy; LDL, low-density lipoprotein; UACR, urine albumin-to-creatinine ratio. 


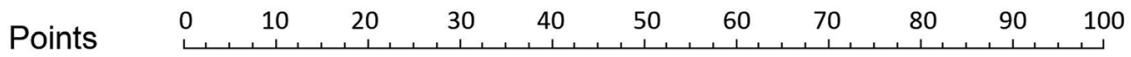

2

4

Stage of UACR

\begin{tabular}{llll}
\hline 1 & 3 & 1 & 5
\end{tabular}

LDL

$\begin{array}{lllllllllllll}1 & 1.5 & 2 & 2.5 & 3 & 3.5 & 4 & 4.5 & 5 & 5.5 & 6 & 6.5 & 7\end{array}$

Total Points

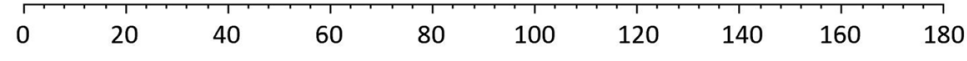

Risk

$\begin{array}{lllllllll}0.05 & 0.1 & 0.2 & 0.3 & 0.4 & 0.5 & 0.6 & 0.7 & 0.8\end{array}$

Figure 4 Nomogram to define the risk for the occurrence of DME. This nomogram was developed according to the statistically significant risk factors (stage of UACR and LDL) (AUC 0.822; 95\% Cl 0.759 to 0.885; $\mathrm{p}<0.001$ ). AUC, area under the curve; DME, diabetic macular oedema; LDL, low-density lipoprotein; UACR, urine albumin-to-creatinine

ratio.

second, calculate the total points: $33+70+34=137$ points and third, link total points to the last row, and a numerical risk incidence will be obtained: about $85 \% .{ }^{30}{ }^{31}$ Using these inexpensive, convenient, fast and intuitive tools, it will be more efficient to identify the patients with T2DM who are at a higher risk of DR and DME. In this way, those patients at increased risks to develop DR or DME can be contrapuntally referred to ophthalmologist, which reduce the waste of medical resources and improve clinical efficiency. It can also alleviate the pressure of screening for DR caused by a huge number of patients with diabetes but insufficient ophthalmologists in China.

There were several limitations in this study. First, this is a retrospective study. The findings in our study needs to be corroborated by prospective studies and randomised controlled trials. Besides, the number of the cases is small. It is better to enlarge the number of the participants.

In conclusion, our study demonstrated that stage of eGFR and UACR were associated with the stage of DR and DME development. The independent risk factors for DR were DM duration, stage of UACR and LDL, while that of DME were stage of UACR and LDL. Therefore, when DR occurs in patients with T2DM, systemic condition such as renal function and blood lipids should be improved as much as possible while treating ocular conditions. Physicians can use the nomogram developed in the current study to estimate the probability of DR and DME in patients with T2DM, thus prompting more efficient referral to ophthalmologists.

Contributors LZ, XZ and DC: conception and design. XZ, YZ, DY, JX and SZ: data collection, analysis and/or interpretation. XZ and DC: drafting the article. HY, JW and JK: revising it critically for important intellectual content. LZ and XZ: final approval of the version to be published. All authors read and approved the final manuscript.

Funding This work was supported by National Natural Science Foundation of China (grant number 81500737) and Guangzhou Science and Technology Programme (grant number 201607010343).

Competing interests None declared.

Patient consent for publication Not required.

Ethics approval This study was approved by the Research Ethics Committee of Guangdong Provincial People's Hospital (registration number: gdrec2016232A).

Provenance and peer review Not commissioned; externally peer reviewed.
Data availability statement Extra data can be accessed via the Dryad data repository at http://datadryad.org/ with the doi: 10.5061/dryad.6kg1sd7.

Open access This is an open access article distributed in accordance with the Creative Commons Attribution Non Commercial (CC BY-NC 4.0) license, which permits others to distribute, remix, adapt, build upon this work non-commercially, and license their derivative works on different terms, provided the original work is properly cited, appropriate credit is given, any changes made indicated, and the use is non-commercial. See: http://creativecommons.org/licenses/by-nc/4.0/.

\section{REFERENCES}

1. Resnikoff S, Pascolini D, Etya'ale D, et al. Global data on visual impairment in the year 2002. Bull World Health Organ 2004;82:844-51.

2. Xu Y, Wang L, He J, et al. Prevalence and control of diabetes in Chinese adults. JAMA 2013;310:948-59.

3. Liu Y, Song Y, Tao L, et al. Prevalence of diabetic retinopathy among 13473 patients with diabetes mellitus in China: a cross-sectional epidemiological survey in six provinces. BMJ Open 2017;7:e013199.

4. Barrett EJ, Liu Z, Khamaisi M, et al. Diabetic microvascular disease: an endocrine Society scientific statement. J Clin Endocrinol Metab 2017;102:4343-410.

5. Thomas MC, Brownlee M, Susztak K, et al. Diabetic kidney disease. Nat Rev Dis Primers 2015;1.

6. Sabanayagam C, Foo VHX, Ikram MK, et al. Is chronic kidney disease associated with diabetic retinopathy in Asian adults? $\mathrm{J}$ Diabetes 2014;6:556-63.

7. Man REK, Sasongko MB, Wang JJ, et al. The association of estimated glomerular filtration rate with diabetic retinopathy and macular edema. Invest Ophthalmol Vis Sci 2015;56:4810-6.

8. Rodríguez-Poncelas A, Mundet-Tudurí X, Miravet-Jiménez S, et al. Chronic kidney disease and diabetic retinopathy in patients with type 2 diabetes. PLoS One 2016;11

9. Romero-Aroca P, Baget-Bernaldiz M, Navarro-Gil R, et al. Glomerular filtration rate and/or ratio of urine albumin to creatinine as markers for diabetic retinopathy: a ten-year follow-up study. J Diabetes Res 2018;2018:1-9.

10. Alberti KG, Zimmet PZ, Definition ZPZ. Definition, diagnosis and classification of diabetes mellitus and its complications. Part 1: diagnosis and classification of diabetes mellitus provisional report of a who consultation. Diabet Med 1998;15:539-53.

11. Wilkinson CP, Ferris FL, Klein RE, et al. Proposed International clinical diabetic retinopathy and diabetic macular edema disease severity scales. Ophthalmology 2003;110:1677-82.

12. Kinyoun J, Barton F, Fisher M, et al. Detection of diabetic macular edema. Ophthalmoscopy versus photography--Early Treatment Diabetic Retinopathy Study Report Number 5. The ETDRS Research Group. Ophthalmology 1989;96:746-50.

13. Levey AS, Stevens LA, Schmid $\mathrm{CH}$, et al. A new equation to estimate glomerular filtration rate. Ann Intern Med 2009;150:604-12.

14. Levey AS, Cattran D, Friedman A, et al. Proteinuria as a surrogate outcome in CKD: report of a scientific workshop sponsored by the National kidney Foundation and the US food and drug administration. Am J Kidney Dis 2009;54:205-26. 
15. lasonos A, Schrag D, Raj GV, et al. How to build and interpret a nomogram for cancer prognosis. J Clin Oncol 2008;26:1364-70.

16. Chen $\mathrm{Y}-\mathrm{H}$, Chen $\mathrm{H}-\mathrm{S}$, Tarng D-C. More impact of microalbuminuria on retinopathy than moderately reduced GFR among type 2 diabetic patients. Diabetes Care 2012;35:803-8.

17. Kramer HJ, Nguyen QD, Curhan G, et al. Renal insufficiency in the absence of albuminuria and retinopathy among adults with type 2 diabetes mellitus. JAMA 2003;289:3273-7.

18. Hammes H-P, Welp R, Kempe H-P, et al. Risk factors for retinopathy and DME in type 2 Diabetes-Results from the German/Austrian DPV database. PLoS One 2015;10:e0132492.

19. Young A, Garg AX. It's about time: extending our understanding of cardiovascular risk from chronic kidney disease. J Am Soc Nephrol 2009;20:2486-7.

20. Rifkin DE, Katz $\mathrm{R}$, Chonchol $\mathrm{M}$, et al. Albuminuria, impaired kidney function and cardiovascular outcomes or mortality in the elderly. Nephrol Dial Transplant 2010;25:1560-7.

21. Zhu F-X, Wu H-L, Tu K-S, et al. Serum levels of copeptin are associated with type 2 diabetes and diabetic complications in Chinese population. J Diabetes Complications 2016;30:1566-70.

22. Sekioka R, Tanaka M, Nishimura T, et al. Serum total bilirubin concentration is negatively associated with increasing severity of retinopathy in patients with type 2 diabetes mellitus. J Diabetes Complications 2015;29:218-21.

23. Klein R, Sharrett AR, Klein BEK, et al. The association of atherosclerosis, vascular risk factors, and retinopathy in adults with diabetes : the atherosclerosis risk in communities study. Ophthalmology 2002;109:1225-34.

24. van Leiden HA, Dekker JM, Moll AC, et al. Blood pressure, lipids, and obesity are associated with retinopathy: the hoorn study. Diabetes Care 2002:25:1320-5

25. Miljanovic B, Glynn RJ, Nathan DM, et al. A prospective study of serum lipids and risk of diabetic macular edema in type 1 diabetes. Diabetes 2004;53:2883-92.

26. Sasaki M, Kawasaki R, Noonan JE, et al. Quantitative measurement of hard exudates in patients with diabetes and their associations with serum lipid levels. Invest Ophthalmol Vis Sci 2013;54:5544-50.

27. Kang EY-C, Chen T-H, Garg SJ, et al. Association of statin therapy with prevention of Vision-Threatening diabetic retinopathy. JAMA Ophthalmol 2019;137:363

28. Du M, Wu M, Fu D, et al. Effects of modified LDL and HDL on retinal pigment epithelial cells: a role in diabetic retinopathy? Diabetologia 2013;56:2318-28.

29. Balachandran VP, Gonen M, Smith JJ, et al. Nomograms in oncology: more than meets the eye. Lancet Oncol 2015;16:e173-80.

30. Liu Y, Yang J, Tao L, et al. Risk factors of diabetic retinopathy and sight-threatening diabetic retinopathy: a cross-sectional study of 13 473 patients with type 2 diabetes mellitus in mainland China. BMJ Open 2017;7:e016280.

31. Semeraro F, Parrinello G, Cancarini A, et al. Predicting the risk of diabetic retinopathy in type 2 diabetic patients. J Diabetes Complications 2011;25:292-7. 